\title{
HOW AND WHY WE LEARN.
}

\section{II.}

In my last article I endeavored to make clear that all of the language used to express metaphysical notions is borrowed from the physical world by analogy. For many reasons this is quite unfortunate, though it has its good side. Undoubtedly to primitive man just beginning to realize that he had a mind and that there were ideas and truths not belonging to the physical world, an independent vocabulary for metaphysics would have been impossible. It was only by the use of figures that he could explain in the vaguest way his dim metaphysical notions. The spirit seemed most like wind; the resemblance was closer than any other that he could imagine; and so he called it "spirit."

Out of these analogies grows poetry; but a figure ceases to be poetic when it becomes so common that the image is not brought before the mind by its use ; and here comes the danger of metaphysics. We go on using the old figurative language when often the figures have lost their force, and yet we are not entirely free from the influence of the figures; there is, subconsciously at least, the notion which they suggest. Hence arise so much of the confusion and even the discussion which have marred and marked the field of metaphysics.

When a figure is used in poetry, no one thinks of pursuing it to the bitter end ; it suggests a likeness sometimes very faint ; it brings a picture before the mind and then passes out, but in metaphysics the figure is pursued as if it were a perfect and exact description. Now, analogy is not identity or even equivalence, and any analogy carried beyond its legitimate application runs into absurdity; particularly is this the case if the analogy be of a lower to a higher object, as of a material fact to a spiritual truth.

It has been pointed out by Dr. Harris and others that the 289 
use of the biologiical analogy - that is, speaking of the mind in terms of life and of education as a vital process like growth has its dangers. Of course it has. No one will deny a certain manifest absurdity if the analogy is carried too far.

It would be easy enough for any idiot to make fun of the comparison of a potato nourishing a body and a truth nourishing a mind. We put a potato into the stomach and it ceases to be a potato, is resolved into its elements, enters into new combinations, and ceases to have independent existence; while a truth is always a truth, and when learned is not destroyed. Yet in this seeming absurdity there is a closer and deeper analogy than at first appears.

The potato is not lost, but modified; also a truth is modified by the receiving mind; for, after all, truth is relative. No truth is the same to all minds or even to two minds, the simplest statement of fact producing a different impression upon different minds and different results as to the thing believed or known. That is, in one sense there is no absolute truth, but all truth is analyzed in the alembic of the knowing mind.

You will recall Dr. Holmes's John and Thomas: "When John and Thomas for instance are talking together, it is natural enough that among the six there should be more or less confusion and misapprehension."

Surely from all points of view the analogy of the mind to living things is infinitely more apt than that of the mind to things without life, and to speak of education as a process of growth through nutrition is certainly vastly nearer the truth than to speak of it as a process of filling a receptacle. Indeed, we might almost reverse the analogy. What lives if not the mind? Who can say that there is any life more real than the life of the mind? Do we not all believe that the only real, permanent, and genuine life is spiritual life, and that hence no analogy could be strictly apt except the biological ?

It is quite evident that schools have, too many of them, based their work upon the physical analogy. Minds have been treated, not as living organisms, but as vacant receptacles. Consider some of the theories underlying school management. 
We say that the children go to school to learn, and so they do. What does learning mean to those who send them to school and to those who teach them? Probably to the majority it means getting knowledge, producing what we commonly call "intelligence." This intelligence includes acquaintance with the common symbols used in reading, writing, and computing. It is a necessary foundation for any real educational work and is recognized as a sort of sub-stratum of all learning.

I will speak a little later of the character of the school which such a view of learning has produced, but it is evicient that growth through nutrition does not enter the consideration of those who plan and conduct the common intelligence schools. Here plainly the mind is a receptacle into which is to be put a limited amount of useful information, to be there preserved against a rainy day. Some, still preserving the analogy of the receptacle, would greatly enlarge the body of knowledge, that is, would have a bigger receptacle filled by a greater number of facts.

As stated in the Report of the Committee of Fifteen, the purpose of school-teaching is to acquaint the child with the civilization in which he is to live; that is, to give him larger intelligence. Note carefully that mention is not made of growing into efficiency in this civilization, but merely of charging the mind with facts relating to it; the knowledge is an extraneous thing poured in. There is no suggestion of vital connection. This view, however, of the purpose of learning is a nobler one than the first, for it greatly enlarges the field of knowledge, but it manifestly defies any definition of education which its advocates would accept. It makes the norm the acquisition of knowledge, as if knowledge were a concrete, extraneous something, and not the effect on the mind, the growth of the mind itself. These theories accept unreservedly a physical, as distinct from a biological, analogy, for the educational processes.

There is another somewhat peculiar view of the object of learning, involving also its method, which has been the basis of whole systems of education; it is the so-called disciplinary view. It squints toward the higher biological view, but that is 
all. It involves a strange mixture of figures. The mind is something to be trained to act in fixed narrow lines, as monkeys and horses are trained. This is the squint toward the biological view. It is also a tool to be sharpened while knowledge is the whetstone. This is the squint away from the biological view toward materialism.

This notion tends to the disregard of content and to the con. sideration of disciplinary value alone. It makes no difference what is studied so long as it furnishes discipline. Any old thing will do. The educational system based upon it is mediæval and theological in origin. Its type of efficient reasoning is that acephalous amusement of the schoolmen, the syllogism, through which a man sitting upon a pedestal may learn the world with his eyes shut. Such training should produce at its best a trick animal, at its worst a saw.

There is still another notion of learning involving both its end and its method, and it rests frankly upon the biological analogy. It is that learning is growth through nutrition, and that the business of the school is to furnish nutriment and exercise to the growing mind. It accepts without questioning the thought that truth is relative, and that it does not exist apart from the mind, of which it is a constituent part; that, when a truth is known, two things are modified, the mind and the truth as stated. The mind that knows a new truth can never be the same mind it was before; it has taken new elements into its being which are bound to affect it for good or evil without end. The statement of truth is necessarily modified by the apperceiving mind. No truth is the same in two minds.

Let us consider the kinds of schools that have been produced by these notions of the method and character of learning. The first or common intelligence notion is responsible for the poorest of all our schools, the school of the three R's and the so-called commercial school or the school of the one R, 'rithmetic. These schools represent the bare bones of education; it is scarcely education at all. They represent the misery of starved minds and are the cause of millions of unhappy failures. Occasionally 
some lover of the past arises and attempts to apotheosize these schools of the three R's, but the people know better and we are getting away from them as rapidly as possible, though, alas, there are too many of them yet. Such schools, so povertystricken, so wholly inadequate for the training of citizens, are the products of the false analogy, the physical as distinguished from the biological.

The larger intelligence notion has caused a distinctly better school because the range of knowledge is broader, its content is richer, and hence the interest of the student is greater. It is the school of verbal culture, in which a great deal is learned about many things. It is at its best in its dealings with history and literature, and at its worst in its study of nature and social life. For it have been written innumerable books about things nature books ; art books ; histories of literature dealing carefully with the dates of the births and the deaths of authors; histories of the world, containing long lists of dates and a few facts appended like tails to kites; information books on all subjects; in short, every possible substitute for real knowledge. It is the school of words, words, words.

But as the subjects dealt with are many and those generally recognized as important, it has inevitably led to higher interests than the first-mentioned school. The mind has been "stored" with much useful knowledge, some of which, in spite of the theory, has nourished it. The advocates of this notion of learning have been friends of higher education as represented in high schools and universities. They have believed the false proverb, "Knowledge is power," and followed the falser notion that knowledge is something that can be stuffed into minds. So naturally, the more stuffing, the more power. It follows, therefore, that they must of necessity be advocates of the greater stuffing machines, high schools and colleges; but such schools, whether elementary or most advanced, have fallen short of the true end of education. They have been foes of freedom, of individual initiative, of self-government, of real, as distinguished from verbal, culture.

The second notion of the proper function of knowledge in 
education, the disciplinary, is responsible for the "formal discipline" fallacy with all its woes. This theory practically disregards the substance of knowledge presented. If the first is responsible for the grad-grind school, the second is responsible for the dull grind which kills the school, crowds out the discouraged, over-accents the law of the survival of the fittest,.and deprives the school of joy and spontaneity, by forcing upon the child a law not his own.

This is serious enough in higher education, but in the elementary school it is deadly. Here it stands for the meaningless repetition of words, for rules without understanding, for language without thought, for drill without content, for life with neither interest nor joy. It is bare, barren, and altogether unlovely. On the ethical side it stands for the breaking of the will, the complete nullification of individual initiative; for absolute, unquestioning, unreasoning submission to external authority; for formalism, hypocrisy, spiritual anarchy; for force and violence. Its ruling motive is the convenience of the autocratic demagog, its scepter the rod employed to enforce the argumentum a posteriori.

Let it be remembered : the great failure in all these schools is the failure to consider the needs of the children, their internal, real, personal needs; their need for food whereon they may grow, as determining to any extent what should be given them. That this must all be determined from without, witness the Report of the Committee of Fifteen. Each child's own mental state may determine the order in which food shall be presented - soup, fish, meat, salad, dessert, but not to even the slightest degree what the food shall be ; for he is not to be nourished, but to be supplied. This is a fatal error.

To be convinced that this statement is not an exaggeration, one needs but to consult any of the standard books on education, not excepting such masterly documents as the Report of the Committee of Ten and the Report of the Committee of Fifteen, especially the latter.

The apperception doctrine of the Herbartians is a partial recognition of the biological nature of education. Of course, the 
writers upon the kindergarten all recognize the mind as living. Aside from these two classes of books the standard text-books upon education, and even upon psychology as applied to education, consider but two objects : the imparting useful information, and disciplining the mind-both ends to be secured through the logical application of established laws foreign to the minds of the children to be taught. Wise teachers have always, to a greater or less extent, sometimes half-consciously, recognized individual need. Theories and systems have seldom done so. The fact that each mind has a law of its own by which it grows; that this law cannot be modified by the application of any extraneous force; that no two minds are alike; that only so much of knowledge is really acquired as is apperceived and assimilated, and that the mind must have nutriment adapted to it - these are facts which, in the abstract, must appeal to all thoughtful teachers, but they are ignored in most plans for the organization of schools and the conduct of school education.

In the next article I shall endeavor to show the character of the true school which consistently treats the mind as a growing organism, and education as a process of growth through nutrition and exercise.

Depaktment of Public Instruction,

C. B. Gilbert.

Rochester, N. Y. 